\title{
X-ray photoelectron spectra and electronic structure of quasi-one-dimensional SbSel crystals
}

\author{
J.Grigas $^{1}$, E.Talik², M.Adamiec² ${ }^{2}$ V.Lazauskas ${ }^{3}$ \\ ${ }^{1}$ Faculty of Physics, Vilnius University, Sauletekio 9/3, LT-10222 Vilnius, Lithuania \\ 2 Institute of Physics, Silesian University, Universytetska 4, 40-007 Katowice, Poland \\ 3 Institute of Theor. Phys. and Astronomy, Vilnius University, Gostauto 12, LT-01108 Vilnius, Lithuania
}

Received September 4, 2006

\begin{abstract}
The paper presents the X-ray photoelectron spectra (XPS) of the valence band (VB) and of the principal core levels from the (110) and (001) crystal surfaces for the quasi-one-dimensional high permittivity SbSel single crystal isostructural to ferroelectric SbSI. The XPS were measured with monochromatized Al Ka radiation in the energy range of $0-1400 \mathrm{eV}$ at room temperature. The VB is located from 1.6 to $20 \mathrm{eV}$ below the Fermi level. Experimental energies of the VB and core levels are compared with the results of theoretical ab initio calculations of the molecular model of the SbSel crystal. The electronic structure of the VB is revealed. Shifts in the core-level binding energies of surface atoms relative to bulk ones, which show a dependency on surface crystallography, have been observed. The chemical shifts of the core levels $(\mathrm{CL})$ in the SbSel crystal for the $\mathrm{Sb}, \mathrm{I}$ and Se states are obtained.
\end{abstract}

Key words: SbSel, electronic structure, XPS

PACS: $79.60 .-i, 82.80 . P v, 87.64 . \mathrm{Lg}$

\section{Introduction}

At present X-ray photoelectron spectroscopy (XPS) is widely used for investigating solids. Due to the reduced co-ordination number, surface atoms have a potential different from the bulk atoms.

Surface CL shifts of the order of $\Delta E_{b} \approx 0.5 \mathrm{eV}$ towards both higher and lower binding energies have been experimentally observed in different materials [1-4]. The magnitude and the sign of the shift depend on electronic and structural properties of the bulk and surface atoms, as well as on a surface structure. XPS studies also revealed a splitting of the CL in the incommensurate phase of the ferroelectric semiconductor $\mathrm{TlInS}_{2}[5]$.

Antimony sulphoiodide, SbSI, is a well-known quasi-one-dimensional ferroelectric semiconductor, which exhibits a number of prominent strongly coupled semiconductive and ferroelectric properties [6]. XPS studies [7] of the SbSI single crystals revealed a huge crystallographic planedependent splitting $(\sim 3 \div 5 \mathrm{eV})$ of the CL due to the different valence state of surface and bulk atoms. Theoretical ab initio calculations confirmed these findings. The valence band (VB) and CL of this quasi-one-dimensional ferroelectric are extremely sensitive to the changes of the chemical environment of atoms.

SbSeI is isostructural to the ferroelectric SbSI [8]. At room temperature, SbSeI crystals possess a high ionic dielectric permittivity $(\varepsilon \approx 300)$, which increases with the temperature decrease [6]. However, due to a higher atomic form factor, i.e. higher density of electrons of Se in SbSeI than of $\mathrm{S}$ in SbSI, the SbSeI crystals are not ferroelectrics in the whole temperature range [9].

The purpose of this paper is to study XPS of the isostructural to SbSI non-ferroelectric SbSeI crystal, to discuss the form and the electronic structure of the VB and to reveal how the CL splitting is related to different valence states of the surface and bulk atoms, and to the quasi-onedimensionality of these crystals. 


\section{Experimental}

The SbSeI crystals were grown from a melt. The obtained crystals were $20 \mathrm{~mm}$ long and $5 \times 5 \mathrm{~mm}^{2}$ in area. They are composed of the chains along the [001] axis. The XPS valence band and the principal core level spectra were measured with monochromatic Al Ka radiation (1486.6 eV) using PHI 5700/660 Physical Electronics Spectrometer. The photoelectron spectra as a function of kinetic energy were analysed in the energy range of $0-1400 \mathrm{eV}$ using a hemispherical mirror analyser with an energy resolution of about $0.3 \mathrm{eV}$. The FAT mode of the spectrometer was used - the pass energy of the analyzer is held at a constant value and the transfer lens system retards the given kinetic energy channel to the range accepted by the analyzer. The measurements were performed on the (110) (broken lengthwise) and (001) (broken crosswise) crystal surfaces cleaved in vacuum, in the low $10^{-10}$ torr range in order to obtain a clean surface. The surface stoichiometry and quality of the both (110) and (001) cleavage planes were checked by measuring the characteristic XPS spectrum (figure 1). The only contaminant was found to be carbon. No other spectral features showing non-stoichiometry or impurities have been found. The size of the $\mathrm{x}$-ray spot was about several square microns. The angle between the X-ray source and the analyzer was $90 \mathrm{deg}$. The electron emission angle, measured from the sample normal, was $45 \mathrm{deg}$. Carbon C $1 \mathrm{~s}(284.5 \mathrm{eV})$ peak was applied for calibration.

While investigating the XPS, it is important to determine the relative concentrations of various constituents. The Multipak Physical Electronics program enables us to perform quantification of the XPS spectra utilizing peak area and peak height sensitivity factors (5.334 for Sb 3d; 0.821 for Se 3d; 6.303 for I 3d). The standard atomic concentration calculation provides a ratio of each component to the sum of the other taken into account the elements in the data. Only those elements for which the specific line is clearly visible in the spectrum are considered. For those lines the background is subtracted using the Shirley method. The limit of the region of the line is individually selected and then the integration is done. The peak shapes were fitted after a background subtraction, using the Voigtian function.

\section{Ab initio calculation of the energy levels, bond order and the charge of atoms}

The method of calculation is based on Hartree-Fock-Roothaan (HFR) equations in the Linear Combinations of Atomic Orbitals (LCAO) approximation for the molecular orbitals (MO). According to the Koopmans' theorem, the one-particle energies obtained from the canonical HRF equations correspond to the approximate energies of the ionisation potential. The molecular orbital (MO) is a linear combination of atomic orbitals $(\mathrm{AO})$. The MO $\left(\phi_{i}\right)$ can be expanded by the AO $\left(\chi_{\mu}(\mathbf{r})\right)$ base:

$$
\phi_{i}(\mathbf{r})=\sum_{\mu=1}^{M} C_{i \mu} \chi_{\mu}(\mathbf{r}),
$$

where $\mu$ is the number of the AO. The $\mathbf{C}$ matrix is obtained by solving Hartree-Fock matrix equation

$$
\mathbf{F C}=\mathbf{S C} \boldsymbol{\varepsilon} .
$$

The method of solving equation (2) as well as the binding energy, bond strength and atomic charge calculation is described in [7]. It is supposed that the electron correlation energy is small. Also, the quantum mechanical calculations do not lokalize the surface states. The calculations were performed using the GAMESS program [10].

\section{Results of the XPS measurements}

Figure 1 shows the XPS of the SbSeI crystal in the energy range from 0 to $1400 \mathrm{eV}$ below the Fermi level without contamination with any gas and only with a small amount of carbon (C 1s peak at $284.5 \mathrm{eV})$. 


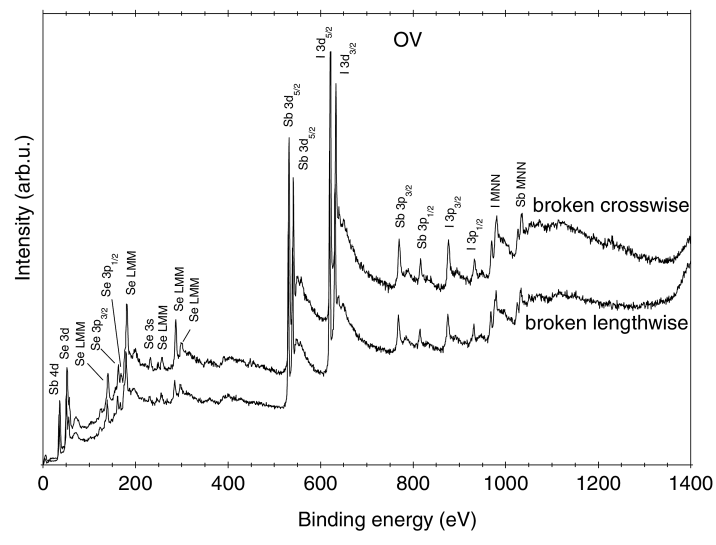

Figure 1. XPS spectrum of SbSeI in the energy range 0 to $1400 \mathrm{eV}$.

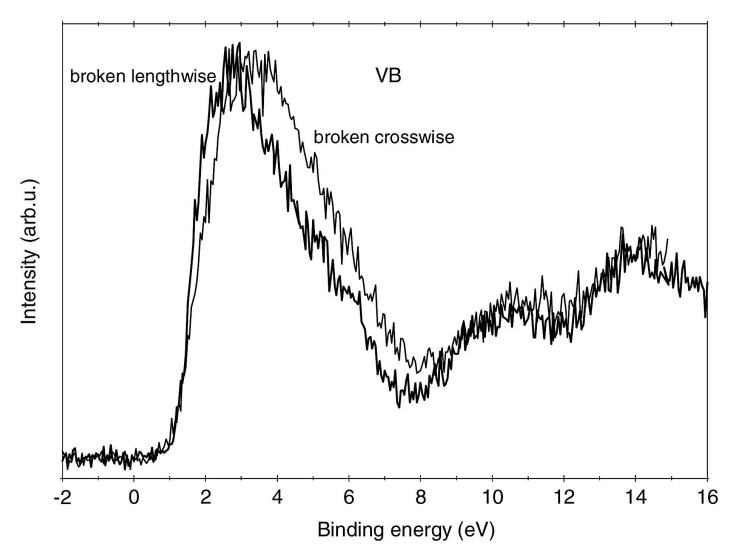

Figure 2. XPS of SbSeI crystal valence band.

Auger spectra of Se LMM as well as of Sb MNN and of I MNN are also seen. The background is caused by secondary electrons. XPS did not show any traces of impurities. Only the carbon was visible after the sample was cleft under ultrahigh vacuum conditions, in the low $10^{-10}$ torr range. We did not find noticeable change of the surface composition with time at fixed temperature as well as with illumination time. The strongest peaks of Sb 3d, Sb 4d, I 3d, I 4d and Se 3d were chosen to investigate the peculiarities of the crystallographic plane-dependent core-level XPS in this quasi-one-dimensional semiconductor.

Figure 2 shows the valence band (VB) spectrum. The spectrum is referred to the Fermi level $\left(\mathrm{E}_{\mathrm{F}}\right)$. The $\mathrm{E}_{\mathrm{F}}$ was defined with the accuracy of $0.3 \mathrm{eV}$. The VB is separated by the gap of about $1.2 \mathrm{eV}$ from the Fermi level and it is located to about $20 \mathrm{eV}$ below $\mathrm{E}_{\mathrm{F}}$. Above $16 \mathrm{eV}$, the $\mathrm{VB}$ is hidden by the $\mathrm{Sb} 4 \mathrm{~d}$ band. In these crystals, the optical band gap is about $1.6 \mathrm{eV}$ at $300 \mathrm{~K}$. It nearly corresponds the value obtained from the XPS measurements. The shape of VB is crystallographic-plane dependent and it will be discussed in section 6 .

Figure 3 shows the spectrum of the nearest to VB spin-orbit doublet of $\mathrm{Sb} 4 \mathrm{~d}$ from the planes broken lengthwise (110) and crosswise (001). The core-level binding energy is referred (in all figures) to the Fermi level with the calibration accuracy of $0.3 \mathrm{eV}$. The peaks of $\mathrm{Sb} 4 d_{5 / 2}$ and $4 \mathrm{~d}_{3 / 2}$ are located at about 33.8 and $35 \mathrm{eV}$, respectively. The binding energy increases $1.7 \mathrm{eV}$ in comparison with $E_{b}$ in pure $\mathrm{Sb}\left(E_{b}=32.1 \mathrm{eV}\right.$ for $4 \mathrm{~d}_{5 / 2}$ and $E_{b}=33.3 \mathrm{eV}$ for $\left.4 \mathrm{~d}_{3 / 2}\right)$.

The peaks of $\mathrm{Sb} 3 \mathrm{~d}_{5 / 2}$ and $3 \mathrm{~d}_{3 / 2}$ are located at about 529.9 and $539 \mathrm{eV}$, respectively (figure 4).

The shift of the binding energy is equal to $1.6 \mathrm{eV}$ in comparison with pure $\mathrm{Sb}\left(E_{b}=\right.$ $528.3 \mathrm{eV}$ for $3 \mathrm{~d}_{5 / 2}$ and $E_{b}=537.4 \mathrm{eV}$ for $\left.3 \mathrm{~d}_{3 / 2}\right)$. The position, pinned by calibration, of $\mathrm{I} 3 \mathrm{~d}_{5 / 2}$ is

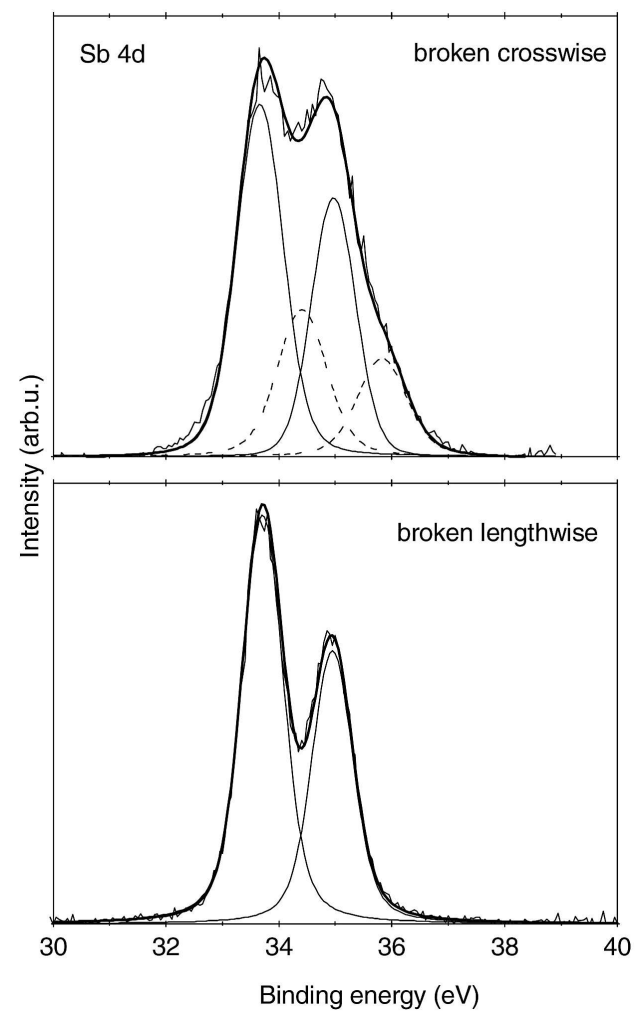

Figure 3. XPS of SbSeI crystal spin-orbit doublet $\mathrm{Sb} 4 \mathrm{~d}$. The chemical shift is $+1.7 \mathrm{eV}$. 
at $E_{b}=619.2 \mathrm{eV}$ and of I $3 \mathrm{~d}_{3 / 2}$ at $E_{b}=630.5 \mathrm{eV}$ (figure 5).

The chemical shift is about $-0.1 \mathrm{eV}$ (broken crosswise) and $-0.2 \mathrm{eV}$ (broken lengthwise) to lower values (for pure I $3 \mathrm{~d}_{5 / 2}$ is at $E_{b}=619.3 \mathrm{eV}$ ), I $3 \mathrm{~d}_{3 / 2}$ is at $E_{b}=630.7 \mathrm{eV}$ ).

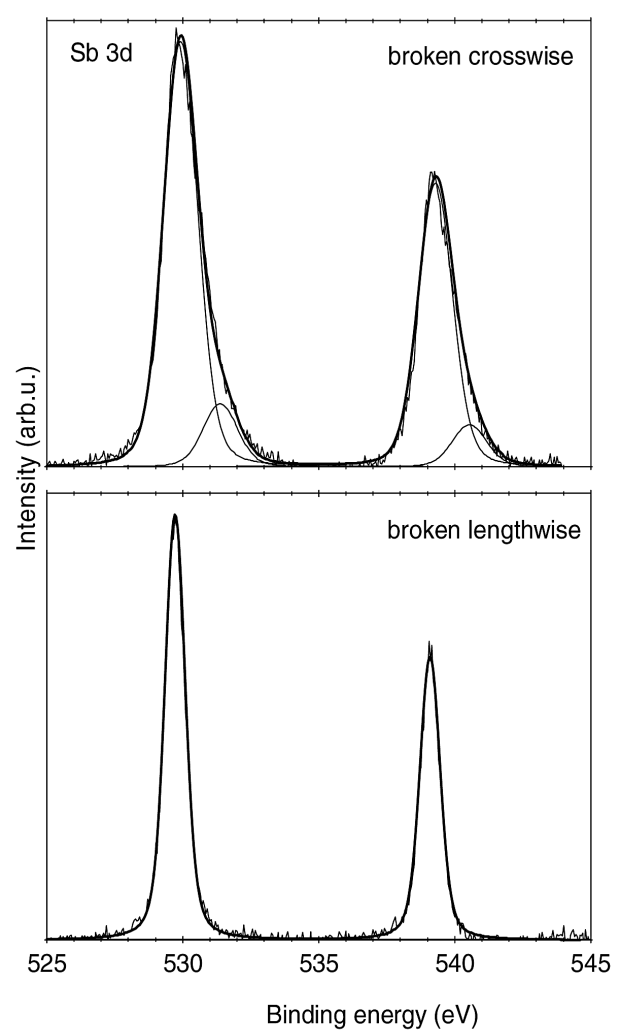

Figure 4. XPS of SbSeI crystal spin-orbit doublet $\mathrm{Sb} 3 \mathrm{~d}$. The chemical shift is $+1.6 \mathrm{eV}$.

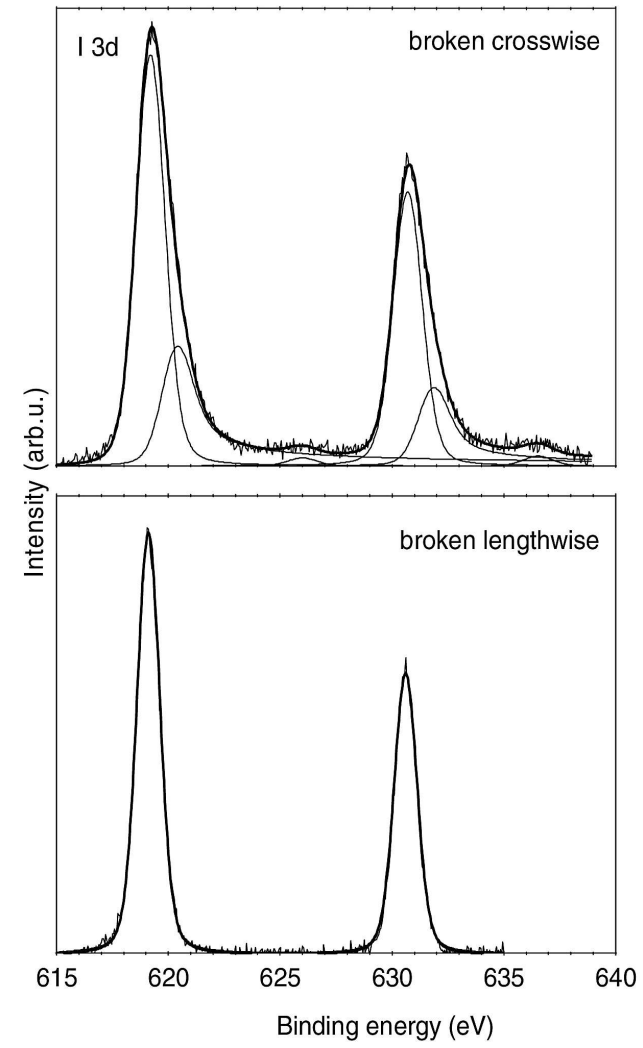

Figure 5. XPS of SbSeI crystal spin-orbit doublet I 3d. The chemical shift is about $-0.1 \mathrm{eV}$ (broken crosswise) and - $0.2 \mathrm{eV}$ (broken lengthwise) sample.

Figure 6 shows the spectrum of the spin-orbit doublet of I $4 \mathrm{~d}$. The peaks of I $4 \mathrm{~d}_{5 / 2}$ and $4 \mathrm{~d}_{3 / 2}$ are located at about 49 and $51 \mathrm{eV}$, respectively. The crosswise spectrum is broader and split into two components.

The position of the overlapping Se $3 \mathrm{~d}_{5 / 2}$ and $\mathrm{Se} 3 \mathrm{~d}_{3 / 2}$ peaks was obtained at $54.5 \mathrm{eV}$ and $55.5 \mathrm{eV}$ (figure 7). For selenium, the chemical shift is also in the opposite direction in relation to $\mathrm{Sb}$, i.e. to the lower binding energy about $-1.1 \mathrm{eV}$ (for pure $\mathrm{Se} 3 \mathrm{~d}_{5 / 2} E_{b}=55.6 \mathrm{eV}$ and for $3 \mathrm{~d}_{3 / 2}$ $\left.E_{b}=56.6 \mathrm{eV}\right)$.

Thus, the electronic structure measurements revealed the chemical shift of Sb states of $+(1.6-$ 1.7) $\mathrm{eV}$ to the higher binding energy, and I states of $-(0.1-0.2) \mathrm{eV}$ and Se states of $-1.1 \mathrm{eV}$ to the lower binding energy. This shift suggests charge transfer from Sb to Se and I - more to Se than to I. In SbSI, the chemical shift of Sb states is of $+1 \mathrm{eV}$ to the higher binding energy, and I states of $-0.8 \mathrm{eV}$ and $\mathrm{S}$ states of $-3 \mathrm{eV}$ to the lower binding energy [7]. Thus, the chemical shifts in SbSI and SbSeI crystals are quite different.

The spin-orbit doublets of all the crosswise spectra from the perpendicular to the chains (001) surface are split into two components, which are clearly seen after resolving the spectra into Gaussian contributions. The split components are separated by about 0.8 to $1.2 \mathrm{eV}$. The larger split was obtained in ferroelectric SbSI crystals [7].

We assign the different components of the split spin-orbit doublets to the surface and bulk 


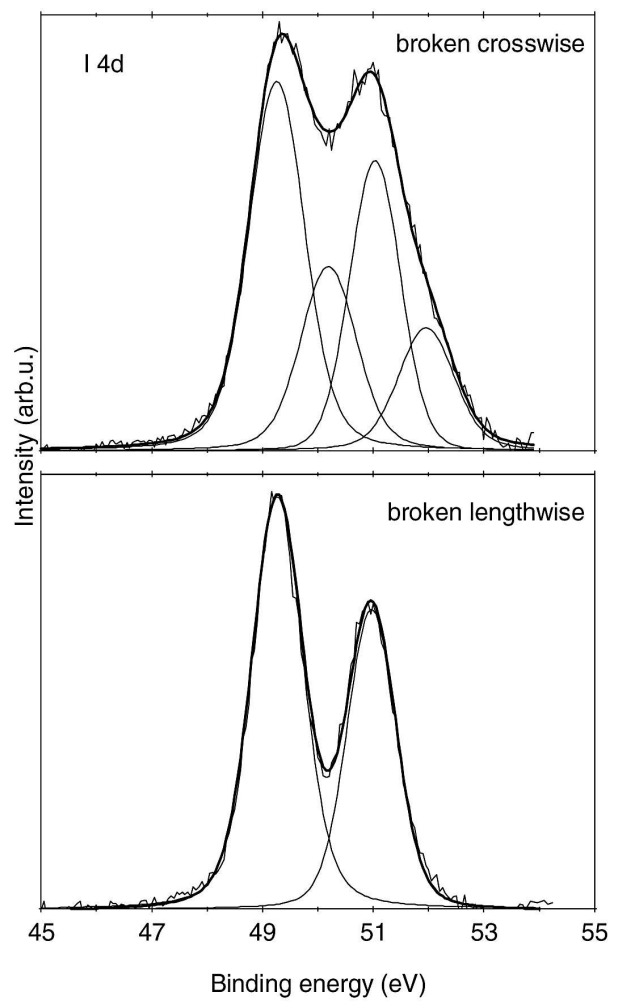

Figure 6. XPS of SbSeI crystal spin-orbit doublet I $4 d$.

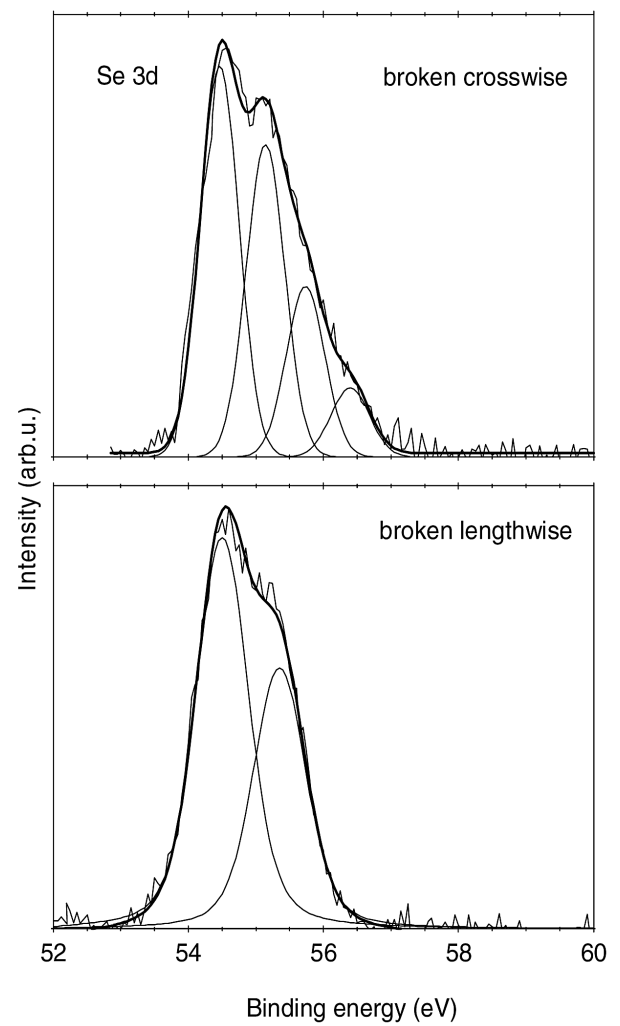

Figure 7. XPS of SbSeI crystal spin-orbit doublet Se 3d. The chemical shift is about $-1.1 \mathrm{eV}$.

contributions. Due to the reasons mentioned in Introduction the core-electron binding energies should be different for surface and bulk atoms. However, the shift in the quasi-one-dimensional SbSeI as well as SbSI [7] crystals is found only for (001) plane-surface. Thus, XPS in SbSeI as well as in ferroelectric SbSI are crystallographic plane-dependent.

\section{Model of SbSel structure and calculated Koopmans' energies}

For the theoretical ab initio calculation of energy levels the molecular model of the SbSeI crystal is needed. The model should be a cluster composed of an even number of molecules. The interaction between the clusters is assumed to be weak.

A projection of the crystal structure of SbSeI on the (110) and (001) planes is shown in figure 8. It contains double chains $\left[(\mathrm{SbSeI})_{\infty}\right]_{2}$ consisting of two chains $(\mathrm{SbSeI})_{\infty}$ related by a two-fold screw axis and linked together by short and strong $\mathrm{Sb}-\mathrm{Se}$ bonds [6,8]. Weak van der Waals-type bonds of $d>3.8 \AA$ (bond strength 0.06 ) bind the double chains. The weakly bonded double chains may be considered as non-interacting and one double chain may be taken as a molecular model of the crystal for the calculation of binding energies. The double chain is formed of many simplified unit cells. Such a simplified unit cell consists of six atoms.

From two to twenty of unit cells such as a molecular model of SbSeI crystal, have been considered for the energy level calculations. Energy levels slightly change with the increase of the cluster. The model of twenty SbSeI molecules is sufficient enough to qualitatively describe the electronic spectrum of the crystal surface and bulk. It was found that the quantum-chemical calculations are sensitive to variation of geometry. 
After breaking the crystal under high vacuum conditions, strong bonds of the (001) surface atoms become open. Due to the reduced coordination number, the (001) surface atoms experience the potential different from the bulk atoms. As a result, the bond strength of the first two atomic layers from the (001) surface change. The bond strength between the first and the second atomic layers doubles at the expense of broken bonds: it increases in the first layer and decreases in the second layer. Löwdin charges mainly change only electronegative atoms in the first three atomic layers from the (001) surface, e.g. they become $\mathrm{Sb}^{+0.59} \mathrm{Se}^{-0.32} \mathrm{I}^{-0.38}$ in the first layer, $\mathrm{Sb}^{+0.59}$ $\mathrm{Se}^{-0.26} \mathrm{I}^{-0.19}$ in the second layer and $\mathrm{Sb}^{+0.57} \mathrm{Se}^{-0.33} \mathrm{I}^{-0.27}$ in the third layer while the charges of all other subsequent atomic layers in bulk are $\mathrm{Sb}^{+0.605} \mathrm{Se}^{-0.31} \mathrm{I}^{-0.29}$. The charges of the surface electronegative atoms compensate electron density of the broken bonds along the [001]-axis. From experimental viewpoint, a surface experiences different concentration of electronegative atoms, e.g. from the XPS of $\mathrm{I} 3 \mathrm{~d}_{5 / 2}$ we have found that the atomic concentration of $\mathrm{I}$ is 35.1 at $\%$ in the broken crosswise crystal while it is 32.9 at \% in the broken lengthwise crystal. The concentration of $\mathrm{Sb}$ is nearly the same in crosswise and lengthwise planes. However, the core-level XPS do not split and are not sensitive to the broken weak bonds along the $\mathrm{x}$ - and $\mathrm{y}$-directions.

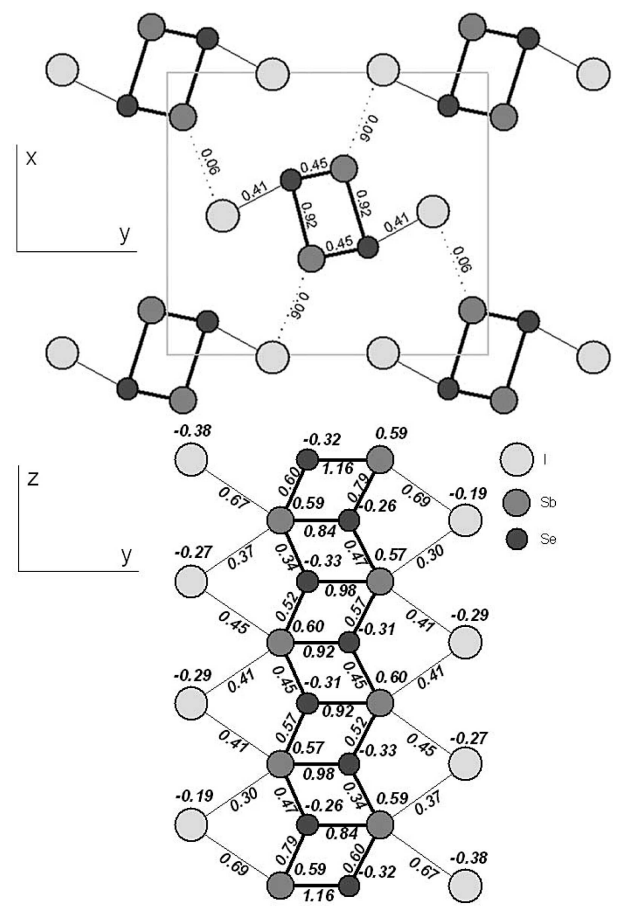

Figure 8. Crystal structure of SbSeI projected on the (001) plane (upper part). The simplified unit cell is formed by two molecules of SbSeI, i.e. by 6 atoms. Molecular chain of SbSeI crystal cluster in the (100) plane (lower part). Dotted line shows the xy-projection of the simplified unit cell. The calculated bond strengths and atom's charges are shown in the picture. They are different in different planes from the surface and reveal in the VB electronic structure and core-level splitting.
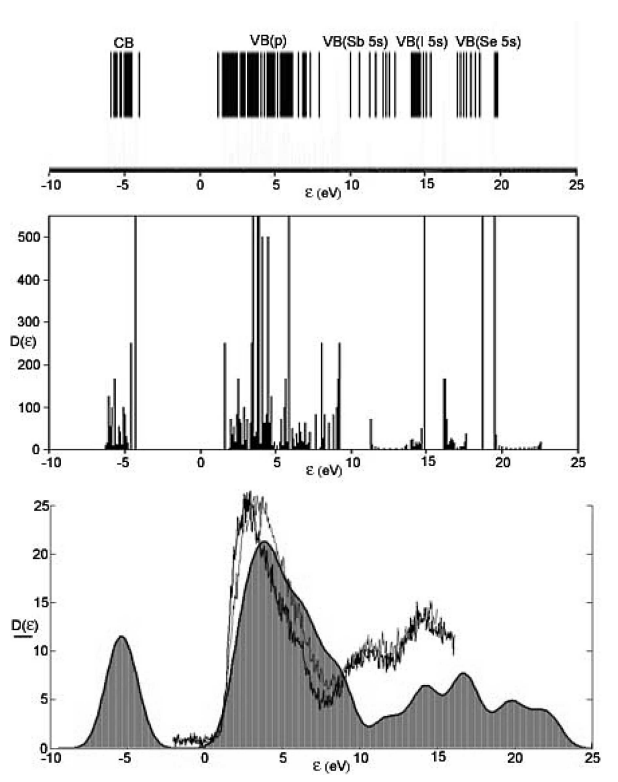

Figure 9. Electronic structure of VB in SbSeI crystal. Representation for the peaks of the density of states and intensity of the valence electrons for 16 molecules SbSeI cluster (top and middle); VB spectra approximation by the Gaussian broadening method and their comparison with experimental XPS (bottom). Energy states broadening parameter is $=0.54 \mathrm{eV}$.

Table 1 presents the theoretical values of the binding energies, calculated with MINI basis set without taking into account the spin-orbit interaction, and experimentally obtained energies, $E_{b \text { exp }}$. The quantum-mechanical method and the chosen model give negative core-level energies higher than their experimental values are. Nevertheless, the model reflects well the electronic structure of the crystal. 
Table 1. Theoretical and experimental values of 20 (SbSeI) molecules chain binding energies at $300 \mathrm{~K}($ in $\mathrm{eV})$.

\begin{tabular}{|c|c|c|}
\hline State & $-E_{b \min }-E_{b \max }$ & $-E_{b(\exp )}(5 / 2-3 / 2)$ \\
\hline Sb 3s & $909-910$ & \\
I 3p & $888-891$ & \\
Sb 3p & $786-787$ & $619-631$ \\
I 3d & $646-649$ & $530-539$ \\
Sb 3d & $558-559$ & \\
Se 3s & $234-23$ & \\
I 4s & $194-190$ & \\
Se 3p & $172-180$ & \\
Sb 4s & $162-163$ & \\
I 4p & $139-142$ & $54.5-55.5$ \\
Sb 4p & $117-119$ & $34-35$ \\
Se 3d & $60-62$ & hidden \\
I 4d & $55-58$ & $13-16$ \\
Sb 4d & $42.5-43.5$ & $1.2-8$ \\
VB Se 4s & $18.5-22.5$ & \\
(s-band) I 5s & $14.5-17.5$ & \\
VB [Se 4p, Sb 5p, I 5p] & & \\
(p-band) & $1.2-9.3$ & \\
\hline
\end{tabular}

\section{Discussion}

Theoretical HFR calculations give the ionization potential with its zero at the vacuum level. The experimental binding energies are referred to the Fermi level. As the energy gap of SbSeI is $1.6 \mathrm{eV}$, in order to compare the experimental and theoretical binding energies and refer to the Fermi level we decreased the calculated ionization potential by the work function $\phi=6.5 \mathrm{eV}$. Theoretical calculations qualitatively explain the experimental values of XPS. Nevertheless, the theoretical eigenvalues of the VB and CL are higher than the experimental binding energies (table 1).

Some discrepancy takes place due to i) a limited basis set of AO for obtaining a molecular orbital solution is used (20 SbSeI molecules as the crystal model), and ii) screening effects are not taken into account. Despite the Koopmans' theorem provides an invaluable tool in assigning XPS it neglects the relaxation processes.

Calculations of the $\mathrm{C}_{i \mu}$ coefficients (equation (1)) shows that VB consists of s- and p-bands separated by $2 \mathrm{eV}$ gap. The longer is the chain the smaller is the gap between the s- and p-bands. The VB is located 1.6 to about $20 \mathrm{eV}$ below the Fermi level. Figure 9 shows theoretically calculated VB form and electronic structure of SbSeI crystal. The spectrum is referred to the Fermi level $\left(\mathrm{E}_{\mathrm{F}}\right)$. The intensities of the XPS were described in three ways (see [11]): a) by the energy states band $\varepsilon_{i}$ from the characteristic equation (2); b) by the peaks of the density of states, and c) by the Gaussian broadening method.

Figure 9 gives the comparison of the earlier discussed calculated SbSeI VB structure with the experimental XPS spectrum up to16 eV. At higher energies the VB overlaps with Sb 4d core level energy (figure 1) and is not shown in figure 9. Intensity of the bands depends on the density of states $\mathrm{D}(\varepsilon)$. The top of figure 9 shows the representation of $\mathrm{D}(\varepsilon)$. Experimental XPS is the integral picture of all electronic states. In the bottom of figure 9 the approximation of SbSeI bands spectra by the Gaussian broadening method and their comparison with experimental XPS is presented.

The calculations give a fairly good description of the places and the widths of the VB energy bands. The VB of SbSeI is composed of the s- and p-type bands separated by $2 \mathrm{eV}$ forbidden gap. The s-band is formed of the Sb 5s, Se 4s, and I $5 \mathrm{~s}$ bands of SbSeI cluster, separated by narrow gaps. Extending a chain of atoms in cluster, an interval between the I 5s and Sb 5s narrows and, finally, disappears. However, the break between the Se 4s and I 5s states remains stable and equal to 1.15 
$\mathrm{eV}$. Among these bands, formed of the electronegative atom states, comes to light the degenerate Se-level which does not mix up with the levels of other atoms of the cluster, while the Sb 5s and I 5 s states are disposed to the mixing.

Table 2. Population (in \%) of VB levels in 4 (SbSeI) molecule cluster. VB consists of s- and p-bands. The energy of levels is denoted by $\varepsilon_{i}$. The last column (D) shows the dominant atomic plane from the (001) surface where photoemission dominates.

\begin{tabular}{|c|c|c|c|c|c|c|}
\hline Band & Atomic state & $-\varepsilon_{1}(e V)$ & $\mathrm{Sb}(\%)$ & $\mathrm{Se}(\%)$ & $\mathrm{I}(\%)$ & $\mathrm{D}$ \\
\hline \multirow[t]{12}{*}{$\mathrm{VB}(\mathrm{s})$} & \multirow[t]{4}{*}{ Se 4s } & 21.6 & 26 & 69 & 5 & $\mathrm{Se}_{2}$ \\
\hline & & 20.2 & 22 & 77 & 2 & $\mathrm{Se}_{2}$ \\
\hline & & 18.5 & 13 & 82 & 5 & $\mathrm{Se}_{1}$ \\
\hline & & 18.4 & 17 & 82 & 1 & $\mathrm{Se}_{1}$ \\
\hline & \multirow[t]{4}{*}{ I $5 \mathrm{~s}$} & 17.2 & 18 & 8 & 74 & $\mathrm{I}_{2}$ \\
\hline & & 16.7 & 6 & 6 & 88 & $\mathrm{I}_{1}$ \\
\hline & & 14.7 & 23 & 8 & 69 & $\mathrm{I}_{1}$ \\
\hline & & 14.6 & 25 & 6 & 70 & $\mathrm{I}_{1}$ \\
\hline & \multirow[t]{4}{*}{$\mathrm{Sb} 5 \mathrm{~s}$} & 13.5 & 55 & 20 & 25 & mixed \\
\hline & & 13.3 & 43 & 10 & 47 & mixed \\
\hline & & 12.1 & 63 & 32 & 5 & mixed \\
\hline & & 10.9 & 53 & 36 & 11 & mixed \\
\hline \multirow{24}{*}{$\operatorname{VB}(\mathrm{p})$} & \multirow[t]{10}{*}{ Se $4 p$} & 8.1 & 31 & 55 & 14 & $\mathrm{Se}_{2}$ \\
\hline & & 7.9 & 36 & 46 & 18 & $\mathrm{Se}_{2}$ \\
\hline & & 7.2 & 43 & 51 & 6 & bulk \\
\hline & & 6.5 & 33 & 41 & 26 & bulk \\
\hline & & 5.9 & 39 & 48 & 13 & bulk \\
\hline & & 5.9 & 38 & 34 & 28 & bulk \\
\hline & & 5.5 & 39 & 39 & 22 & bulk \\
\hline & & 5.3 & 21 & 49 & 30 & bulk \\
\hline & & 5.2 & 27 & 31 & 42 & bulk \\
\hline & & 5.0 & 31 & 34 & 35 & bulk \\
\hline & \multirow{9}{*}{ Sb 5p } & 4.8 & 31 & 45 & 24 & bulk \\
\hline & & 4.1 & 14 & 58 & 28 & bulk \\
\hline & & 4.0 & 22 & 15 & 63 & bulk \\
\hline & & 3.7 & 23 & 18 & 59 & bulk \\
\hline & & 3.5 & 11 & 41 & 38 & bulk \\
\hline & & 3.3 & 1 & 52 & 48 & bulk \\
\hline & & 3.2 & 10 & 43 & 47 & bulk \\
\hline & & 2.7 & 16 & 31 & 53 & bulk \\
\hline & & 2.5 & 9 & 32 & 59 & bulk \\
\hline & \multirow{5}{*}{ I $5 p$} & 2.3 & 3 & 2 & 95 & $\mathrm{I}_{1}$ \\
\hline & & 2.1 & 2 & 1 & 97 & $\mathrm{I}_{1}$ \\
\hline & & 2.0 & 1 & 11 & 88 & $\mathrm{I}_{1}$ \\
\hline & & 1.7 & 14 & 16 & 70 & $\mathrm{I}_{1}$ \\
\hline & & 1.2 & 13 & 21 & 66 & $\mathrm{I}_{1}$ \\
\hline
\end{tabular}

The form of the VB can be explained by the analysis of MO population. Knowing the MO coefficients $\mathrm{C}_{i \mu}$ (equation (1)), one can evaluate the contribution of A atom electrons for $\varepsilon_{i}$ state:

$$
p_{i A}=\sum_{\mu \in A}^{M} C_{0 i \mu}^{2} .
$$

There $\mathbf{C}_{0}=\mathbf{S}^{-1 / 2} \mathbf{C}$ is the matrix of MO coefficients orthogonolized according to Löwdin. The obtained results are shown in table 2, i.e. the population of the VB states for 4 (SbSeI) cluster. 
All binding energies in table 2 and all figures are referred to $\mathrm{E}_{F}$. From the table 2 one can see that the sharp ionization side (near $\mathrm{E}_{F}$ ) of the most intensive p-band of the VB is formed by I atom $(66 \%)$ of the first plane from the surface perpendicular to the z-axis (see figure 8 ) as well as by Se $(21 \%)$ and Sb (14\%) electrons. Since the Sb 5p contribution is small, the VB edge is sharp. The highest MO of the VB is mainly composed of I $5 \mathrm{p}$ of the first surface atomic plane. When the atomic chain is long, this level degenerates. The high-energy p-band side of the VB is formed of the Se 4p (55\%) states of the second plane from the surface, as well as by the Sb $5 \mathrm{p}(31 \%)$ and I 5p (14\%) states. The lower-energy side of the s-band is formed by the Se (36\%), Sb (53\%) and I (11\%) mixed surface and bulk states. The higher-energy side of the s-band is formed by the Se $(69 \%)$ states from the second plane from the surface, as well as Sb $(26 \%)$ and I (5\%) states. There are no states in the VB composed of pure Sb states MO. Interaction of Sb atoms with other atoms increases the width of the VB. Sb atoms also interact with the atoms of the neighbouring chains (figure 8) and also increase the p-band. The conduction band (CB) edge is formed almost $100 \%$ by the Sb 5p states from the first surface plane. Therefore, the donor level is of Sb 5p states and the acceptor level is of I 5 p states.

The form of the VB is well approximated by the Gaussian smearing with $\sigma=0.1$, and this is nearly in accordance with the spectral resolution of the employed X-ray photoelectron spectrometer.

Bond strengths and charges of surface atoms in (001) plane (figure 8) differ considerably from bulk atoms. Bonds become stronger on the surface. The charge of surface Se and I atoms is smaller than of bulk atoms. Breaking of the strong bonds should cause large shifts of the binding energies of the (001) surface atoms. Such changes should cause the splitting of the spin-orbit doublets.

Using the HFR method we have calculated the core-level density of states of surface and bulk atoms. HFR method does not take into account the spin-orbit interaction and instead of spinorbit doublets we have single bands about $3 \mathrm{eV}$ (figures 3-7). The calculated average energies are smaller than the experimental ones (table 1) due to the reasons discussed above. All the CL have the structure similar to VB: electrons of bulk atoms form the middle of the bands, electrons of the first Se and I atoms layer from the (001) surface form the more positive side while electrons of the second atom layer form the more negative side of the Se 3d, I 3d and I 4d bands. Electrons of the second Sb layer from the (001) surface form the more positive side of $\mathrm{Sb} 3 \mathrm{~d}$ and $\mathrm{Sb} 4 \mathrm{~d}$ bands, while the electrons of the first and even of the third layer atoms form the more negative sides.

As the charge of the first and second layer atoms most of all differ from the charge of the bulk atoms, the split of the crosswise spin-orbit doublets may be assigned to the core-level shift by $1-1.2 \mathrm{eV}$ of the surface layer atoms relative to bulk atoms. So, the results presented in figures 3 to 7 may be interpreted as the crystallographic plane-dependent core-level binding energy shifts of surface atoms relative to bulk atoms. However, this shift is smaller than in ferroelectric SbSI [7]. High concentration of electrons or holes is needed for the screening of high ionic polarization (in SbSI also the spontaneous polarization).

\section{Conclusions}

X-ray photoelectron spectra of the valence band and of the principal core levels of the quasione-dimensional semiconductor SbSeI are presented in the energy range from 0 to $1400 \mathrm{eV}$. A molecular model of the crystal is used for ab initio theoretical calculations of the binding energies. The electronic structure of valence band is calculated and confirmed experimentally. The crystal is more ionic according to Mulliken than to Löwdin charges but it is less ionic than the isostructural ferroelectric SbSI crystal. Only three atomic layers from a surface along the [001]-axis may be considered as the surface layers. From the fourth atomic layer, the charges and the bond strength correspond to the bulk. The XPS studies revealed the crystallographic plane-dependent surface atom core-electron binding energy shifts relative to bulk atoms. However, this shift is smaller than in ferroelectric SbSI crystal. 


\title{
8. Acknowledgement
}

This work was performed, in part, within the framework of the Lithuanian-Ukrainian project "Development and studies of new materials for information technologies, acousto-, opto- and nanoelectronics".

\section{References}

1. van der Veen J.F., Himpsel F.J., Eastman D.E. Phys. Rev. Letters, 1980, 44, 189.

2. Kaindl G., Chiang T.-C., Mendel T. Phys. Rev. B, 1983,28, 3612.

3. Chiang T.C., Kaindl G., Mendel T. Phys. Rev. B, 1986, 33, 695.

4. Irizawa A., Yamasaki A., et al. Solid St. Commun., 2002, 124, 1.

5. Grigas J., Talik E. Phys. Status Solidi (b), 2003, 237, 494.

6. Grigas J. Microwave dielectric spectroscopy of ferroelectrics and related materials. Gordon and Breach Science Publ. OPA Amsterdam, 1996, 161-188.

7. Grigas J., Talik E., Lazauskas V. Lithuanian J. Phys., 2004, 44, 427.

8. Vots G.P., Rentreper P.J. Zeitshrift für Krystallography, 1982, 161, 111.

9. Kvedaravicius S., Audzijonis A., Mykolaitiene N., Grigas J. Ferroelectrics, 1996, 177, 181.

10. Schmidt M.W., Baldrige K.K., et all. J. Comput. Chem., 1993, 14, 1347.

11. Lazauskas V., Nelkinas V., Grigas J., Talik E., Gavryushin V. Lithuanian J. Phys., 2006, 46, 205.

\section{Рентгенівські фотоелектронні спектри та електронна структура квазіодновимірних кристалів SbSel}

\author{
Й.Грігас ${ }^{1}$, Е.Талік², М.Адамєц², В.Лазаускас ${ }^{3}$ \\ 1 Фізичний факультет, Вільнюський університет, вул. Саулетекіо, 9/3, LT-10222 Вільнюс, Литва \\ 2 Інститут фізики, Сілезький університет, вул. Університетська, 4, 40-007 Катовіце, Польща \\ 3 Інститут теоретичної фізики і астрономії, Вільнюський університет, вул. Гостауто, 12, LT-01108 \\ Вільнюс, Литва
}

Отримано 4 вересня 2006 р.

\begin{abstract}
Дана стаття представляє рентгенівські фотоелектронні спектри (РФС) валентної зони (ВЗ) і остовних рівнів кристалічних поверхонь (110) і (001) квазіодновимірного монокристалу SbSel з високою проникливістю, який ізоструктурний сегнетоелектрику SbSI. РФС виміряно з використанням монохроматичного випромінювання Al $K \alpha$ в енергетичному діапазоні 0-1400 еВ при кімнатній температурі. В3 розташована від 1.6 до 20 еВ нижче рівня Фермі. Експериментальні енергії В3 та остовних рівнів порівняно з результатами теоретичних розрахунків "з перших принципів" для молекулярної моделі кристалу SbSel. Встановлено електронну структуру ВЗ. Виявлено зсуви остовних енергій зв'язку поверхневих атомів відносно об'ємних, які демонструють залежність від кристалографії поверхні. Отримано хімічні зсуви остовних рівнів у кристалі SbSel для $\mathrm{Sb}$, Se та I.
\end{abstract}

Ключові слова: SbSel, електронна структура, РФС

PACS: $79.60 .-i, 82.80 . P v, 87.64 . \mathrm{Lg}$ 